\title{
Using gene expression profiles from peripheral blood to identify asymptomatic responses to acute respiratory viral infections
}

\author{
Alexander Statnikov ${ }^{1,2^{*}}$, Nikita I Lytkin ${ }^{1}$, Lauren McVoy ${ }^{3}$, Jörn-Hendrik Weitkamp ${ }^{4}$, Constantin F Aliferis ${ }^{1,3,5}$
}

\begin{abstract}
Background: A recent study reported that gene expression profiles from peripheral blood samples of healthy subjects prior to viral inoculation were indistinguishable from profiles of subjects who received viral challenge but remained asymptomatic and uninfected. If true, this implies that the host immune response does not have a molecular signature. Given the high sensitivity of microarray technology, we were intrigued by this result and hypothesize that it was an artifact of data analysis.

Findings: Using acute respiratory viral challenge microarray data, we developed a molecular signature that for the first time allowed for an accurate differentiation between uninfected subjects prior to viral inoculation and subjects who remained asymptomatic after the viral challenge.

Conclusions: Our findings suggest that molecular signatures can be used to characterize immune responses to viruses and may improve our understanding of susceptibility to viral infection with possible implications for vaccine development.
\end{abstract}

\section{Background}

Acute respiratory viral infections cause significant morbidity and mortality in the United States and worldwide. Currently clinicians do not have practical means to make a timely and accurate diagnosis of acute viral respiratory infections and often resort to unnecessary antibiotic treatment, which increases healthcare costs and facilitates development of antibiotic resistance. Recently a novel approach was proposed for the diagnosis of acute respiratory infections based on microarray gene expression profiles from peripheral blood samples from human subjects [1]. Using human viral challenge studies with live human rhinovirus (HRV), respiratory syncytial virus (RSV), and influenza A, Zaas et al. developed an "acute respiratory viral response" 30-gene panviral signature that accurately identified symptomatic patients with viral infection. The same study also reported that gene expression profiles of subjects prior to viral inoculation (at baseline) were indistinguishable

\footnotetext{
* Correspondence: alexander.statnikov@med.nyu.edu

'Center for Health Informatics and Bioinformatics, New York University

School of Medicine, New York, NY 10016, USA

Full list of author information is available at the end of the article
}

from profiles of subjects who received viral challenge but remained asymptomatic and uninfected. Given the high sensitivity of microarray technology, we were intrigued by the latter result and hypothesize that it was an artifact of data analysis. Since the gene expression dataset of Zaas et al. was deposited in the Gene Expression Omnibus, we were able to verify our hypothesis and discover significant differences between these two groups of samples. We demonstrate this by developing a molecular signature that discriminates with high accuracy between uninfected subjects at baseline (prior to viral inoculation) and asymptomatic subjects at the time correlating to peak symptoms in the symptomatic group. This finding has important implications for a better understanding of the complex human immune response to viral antigens. Genes that are differentially expressed in the two groups may provide important clues about decreased susceptibility to viral challenge, which could result in more effective vaccine development or novel therapeutic strategies. 


\section{Methods}

To arrive at our findings, we reanalyzed the gene expression data of Zaas et al. that contains measurements of 22,277 oligonucleotide probes for 56 blood samples from uninfected subjects (measured at baseline, prior to inoculation) and 30 blood samples from subjects who received viral challenge but remained asymptomatic (measured at corresponding peak symptoms time specific for each virus). We used an improved data-analytic protocol that avoids selection of redundant and biologically irrelevant genes and at the same time maximizes predictive accuracy of the signature [2]. This protocol has recently allowed us to develop a highly accurate and compact molecular signature for differentiation between uninfected subjects and those with acute respiratory viral infections. This signature had high reproducibility as evidenced by its nearly perfect accuracy in the independent data of Ramilo et al. [3], and was comprised of genes that are involved in the host immune response [2].

Our data-analytic protocol first involved selection of genes by GLL-PC, a supervised multivariate biomarker discovery method that provably discovers genes in the local pathway around the response variable of interest [4,5]; additional details about GLL-PC are provided in [Additional file 1]. Next, Support Vector Machine (SVM) classifiers with linear kernel and penalty hyperparameter $C=100$ were fitted on the selected genes [6]. In order to obtain an unbiased estimate of predictive accuracy that will hold in applications of this molecular signature on future subjects, gene selection and SVM classifier training were performed by stratified 10-fold cross-validation repeated 100 times for different splits of subject into 10 folds [2,7]. Finally, we ensured signature reproducibility and assessed its statistical significance by using a permutation test with significance level $\alpha=0.05$ and 10,000 permutations [8].

\section{Results and Discussion}

The data-analytic protocol described above yields an unbiased estimate of predictive accuracy $=0.85$ area under ROC curve (AUC); 95\% confidence interval [0.76; 0.94] AUC. On average GLL-PC selected 7 genes depending on the training set of cross-validation. Genes selected by GLL-PC in more than $20 \%$ of the training sets are listed in Table 1. Genes that were selected in a smaller fraction of training sets may be artifacts of small sample size. Next, GLL-PC and SVM were applied on the entire set of samples, resulting in a 6-gene signature comprised of genes EIF2S1, ZNF91, RBM3, ATP5S, TPPP3, GPR97. Note that all these six genes were also among the top seven most frequently selected by GLLPC during cross-validation (Table 1), which demonstrates the stability of this gene set despite the inherent heterogeneity of gene expression across subjects.

Of the eight genes shown in Table 1, four are known to be involved in the host immune response. EIF2S1 encodes the alpha subunit of the translation initiation factor eIF2 complex (eIF2 $\alpha$ ), which initiates protein synthesis. This protein appears to be crucial for the survival of virally infected cells [9]. The increase of expression of EIF2S1 that is observed in the data could mean that host protein synthesis is turned on as response to viral exposure. On the other hand, many viruses, including influenza A viruses, have developed mechanisms favoring the translation of viral over cellular mRNAs leading to a switch from cellular to viral protein synthesis, while inhibiting the cell-encoded antiviral pathways [10]. RBM3 encodes a protein that is a member of the heterogeneous nuclear ribonucleoproteins (hnRNPs) that appears to enhance global protein synthesis [11] but also has a role in viral transcription and replication [12]. The ZNF91 gene family is a subset of the Krüppelassociated box (KRAB)-containing group of zinc finger genes. Zinc-finger proteins containing the KRAB

Table 1 Most frequently selected genes by GLL-PC during cross-validation

\begin{tabular}{lllc}
\hline $\begin{array}{c}\text { Probe set } \\
\text { ID }\end{array}$ & $\begin{array}{c}\text { Gene } \\
\text { symbol }\end{array}$ & Gene name & $\begin{array}{c}\text { Frequency of selection by GLL-PC during cross- } \\
\text { validation }\end{array}$ \\
\hline 201143_s_at & EIF2S1 & eukaryotic translation initiation factor 2, subunit 1 alpha, 35kDa & $99 \%$ \\
\hline 218876_at & TPPP3 & tubulin polymerization-promoting protein family member 3 & $95 \%$ \\
\hline 206059_at & ZNF91 & zinc finger protein 91 & $94 \%$ \\
\hline 208319_s_at & RBM3 & RNA binding motif (RNP1, RRM) protein 3 & $74 \%$ \\
\hline 220404_at & GPR97 & G protein-coupled receptor 97 & $67 \%$ \\
\hline 202124_s_at & TRAK2 & trafficking protein, kinesin binding 2 & $58 \%$ \\
\hline 213995_at & ATP5S & $\begin{array}{l}\text { ATP synthase, H+ transporting, mitochondrial F0 complex, subunit s } \\
\text { (factor B) }\end{array}$ & $32 \%$ \\
\hline 208650_s_at & CD24 & CD24 molecule & $20 \%$ \\
\hline
\end{tabular}

Genes that were also selected by GLL-PC on the entire set of samples are shown with bold. 
domain are transcriptional regulators that have been associated with suppression of viral proliferation [13]. The related transcriptional factor OTK18 has been shown to be induced by and to suppress HIV-1 infection in mononuclear cells [14]. Finally, CD24 is involved in the CD24-Siglec G pathway that protects the host against a fatal reaction to pathological cell death and discriminates danger- versus pathogen-associated molecular patterns [15].

To the best of our knowledge, the remaining 4 genes from Table 1 (TPPP3, GPR97, ATP5S, TRAK2) have not yet been specifically associated with host immune responses, however this does not rule them out as novel pharmaceutical targets. Some of these genes are involved in normal cellular function and for some there is evidence of their association with host immune responses: TPPP3 encodes members of the tubulin family of proteins, and one study has shown an increase of tubulin in influenza infection [16]. GPR97 encodes a member of $\mathrm{G}$ protein-coupled receptors that bind chemokines on the surface of immune cells. Chemokine signaling is critical for effective antiviral immune response activation. ATP5S encodes a subunit of mitochondrial ATP synthase, and it has been suggested that an increase in mitochondrial activity plays a relevant role in viral replication [17]. TRAK2 encodes a trafficking factor that facilitates expression of potassium channels to the cell surface [18].

In further analysis, we identify a likely reason that precluded Zaas et al. from detecting a difference between uninfected subjects at baseline and asymptomatic subjects at corresponding peak time. The study of Zaas et al. used 30 genes from the "acute respiratory viral response" signature (that was designed for differentiation of symptomatic subjects from uninfected individuals) to perform a different classification task. It is therefore not surprising that even though these 30 genes were predictive for diagnosis of symptomatic subjects from uninfected individuals, they were not predictive for differentiating uninfected subjects at baseline from asymptomatic subjects at the time corresponding to peak symptoms in the symptomatic group. We have verified this assertion by running the original data analysis software of Zaas et al. that resulted in random predictive accuracy $(0.50 \mathrm{AUC})$ when using 30 genes from the "acute respiratory viral response" signature and a nontrivial predictive accuracy (0.66 AUC) when using genes selected specifically for the classification task of interest. The remainder of the discrepancy between performance of the protocol of Zaas et al. and one used in the present study is due to the choice of methods for gene selection, classification, and accuracy estimation (crossvalidation). If we substitute sparse probit regression with SVMs in the analysis protocol of Zaas et al., the predictive accuracy increases to 0.75 AUC. If we also substitute factor analysis-based gene selection with GLL-PC and use repeated 10-fold cross-validation, we obtain the result identical to our analysis, i.e. 0.85 AUC.

\section{Conclusions}

In summary, upon reanalysis, the data provided in the study by Zaas et al. [1] demonstrate that there is a difference between gene expression profiles of the uninfected subjects prior to viral exposure and the asymptomatic subjects after the exposure. This observation is important for understanding host immune response and warrants validation in independent gene expression data and/or with RT-qPCR. A more detailed understanding of molecular factors that enable some exposed subjects to avoid infection or remain asymptomatic after the exposure while others demonstrate clinical illness could provide targets for development of more effective vaccines and antiviral treatments. More studies should be undertaken to better characterize the gene expression changes in specific viral infections and patient populations.

\section{Additional material}

Additional file 1: GLL-PC and one of its instantiations - Semi-

interleaved HITON-PC. This file contains a description of the supervised biomarker discovery framework GLL-PC and one of its instantiations, termed Semi-interleaved HITON-PC, that was used in this work.

\section{Acknowledgements}

We thank the authors of [1] for promptly and generously sharing with us their data, software, and details about their analyses. Alexander Statnikov and Constantin F. Aliferis are acknowledging support from grants R56 LM007948-04A1 from the National Library of Medicine, National Institute of Health and 1UL1RR029893 from the National Center for Research Resources, National Institutes of Health. Jörn-Hendrik Weitkamp was supported by the Eunice Kennedy Shriver National Institute of Child Health and Human Development grant K08HD061607. Lauren McVoy was supported by a fellowship from the Physician Scientist Training Program at New York University School of Medicine.

\section{Author details}

${ }^{1}$ Center for Health Informatics and Bioinformatics, New York University School of Medicine, New York, NY 10016, USA. ²Department of Medicine, New York University School of Medicine, New York, NY 10016, USA. ${ }^{3}$ Department of Pathology, New York University School of Medicine, New York, NY 10016, USA. ${ }^{4}$ Division of Neonatology, Department of Pediatrics, Vanderbilt University School of Medicine and Monroe Carell Jr. Children's Hospital at Vanderbilt, Nashville, TN 37232, USA. ${ }^{5}$ Department of Biostatistics, Vanderbilt University, Nashville, TN, 37232, USA.

\section{Authors' contributions}

Conceived and designed the experiments: NIL, AS, CFA. Performed the experiments: NIL, AS. Analyzed the results of experiments: NIL, AS, CFA, JHW, LM. Wrote the paper: NIL, AS, CFA, JHW, LM. All authors read and approved the final manuscript.

\section{Competing interests}

The authors declare that they have no competing interests. 


\section{References}

1. Zaas AK, Chen M, Varkey J, Veldman T, Hero AO, Lucas J, et al: Gene expression signatures diagnose influenza and other symptomatic respiratory viral infections in humans. Cell Host Microbe 2009, 6:207-217.

2. Statnikov A, McVoy L, Lytkin N, Aliferis CF: Improving development of the molecular signature for diagnosis of acute respiratory viral infections. Cell Host Microbe 2010, 7:100-101.

3. Ramilo O, Allman W, Chung W, Mejias A, Ardura M, Glaser C, et al: Gene expression patterns in blood leukocytes discriminate patients with acute infections. Blood 2007, 109:2066-2077.

4. Aliferis CF, Statnikov A, Tsamardinos I, Mani S, Koutsoukos XD: Local Causal and Markov Blanket Induction for Causal Discovery and Feature Selection for Classification. Part I: Algorithms and Empirical Evaluation. Journal of Machine Learning Research 2010, 11:171-234.

5. Aliferis CF, Statnikov A, Tsamardinos I, Mani S, Koutsoukos XD: Local Causal and Markov Blanket Induction for Causal Discovery and Feature Selection for Classification. Part II: Analysis and Extensions. Journal of Machine Learning Research 2010, 11:235-284.

6. Vapnik VN: Statistical learning theory New York: Wiley 1998.

7. Braga-Neto UM, Dougherty ER: Is cross-validation valid for small-sample microarray classification? Bioinformatics 2004, 20:374-380.

8. Aliferis CF, Statnikov A, Tsamardinos I, Schildcrout JS, Shepherd BE, Harrell FE: Factors Influencing the Statistical Power of Complex Data Analysis Protocols for Molecular Signature Development from Microarray Data. PLOS ONE 2009, 4:e4922.

9. Kepp O, Senovilla L, Galluzzi L, Panaretakis T, Tesniere A, Schlemmer F, et al: Viral subversion of immunogenic cell death. Cell Cycle 2009, 8:860-869.

10. Schneider RJ, Mohr I: Translation initiation and viral tricks. Trends Biochem Sci 2003, 28:130-136.

11. Dresios J, Aschrafi A, Owens GC, Vanderklish PW, Edelman GM, Mauro VP: Cold stress-induced protein Rbm3 binds $60 \mathrm{~S}$ ribosomal subunits, alters microRNA levels, and enhances global protein synthesis. Proc Natl Acad Sci USA 2005, 102:1865-1870.

12. Wright CF, Oswald BW, Dellis S: Vaccinia virus late transcription is activated in vitro by cellular heterogeneous nuclear ribonucleoproteins. J Biol Chem 2001, 276:40680-40686.

13. Yang Z, Wood C: The transcriptional repressor K-RBP modulates RTAmediated transactivation and lytic replication of Kaposi's sarcomaassociated herpesvirus. J Virol 2007, 81:6294-6306.

14. Carlson KA, Leisman G, Limoges J, Pohlman GD, Horiba M, Buescher J, et al: Molecular characterization of a putative antiretroviral transcriptional factor, OTK18. J Immunol 2004, 172:381-391.

15. Chen GY, Tang J, Zheng P, Liu Y: CD24 and Siglec-10 selectively repress tissue damage-induced immune responses. Science 2009, 323:1722-1725.

16. Ohman T, Rintahaka J, Kalkkinen N, Matikainen S, Nyman TA: Actin and RIG-I/MAVS signaling components translocate to mitochondria upon influenza A virus infection of human primary macrophages. J Immunol 2009, 182:5682-5692

17. Novoa RR, Calderita G, Arranz R, Fontana J, Granzow H, Risco C: Virus factories: associations of cell organelles for viral replication and morphogenesis. Biol Cell 2005, 97:147-172.

18. Grishin A, Li H, Levitan ES, Zaks-Makhina E: Identification of gammaaminobutyric acid receptor-interacting factor 1 (TRAK2) as a trafficking factor for the K+ channel Kir2.1. J Biol Chem 2006, 281:30104-30111.

doi:10.1186/1756-0500-3-264

Cite this article as: Statnikov et al:: Using gene expression profiles from peripheral blood to identify asymptomatic responses to acute respiratory viral infections. BMC Research Notes 2010 3:264.

\section{Submit your next manuscript to BioMed Central and take full advantage of:}

- Convenient online submission

- Thorough peer review

- No space constraints or color figure charges

- Immediate publication on acceptance

- Inclusion in PubMed, CAS, Scopus and Google Scholar

- Research which is freely available for redistribution

Submit your manuscript at www.biomedcentral com/submit
Biomed Central 